\title{
Mechanical Degradation Analysis of an Amorphous Silicon Solar Module
}

\author{
Gilbert Osayemwenre *(i) and Edson Meyer \\ Fort Hare Institute of Technology (FHIT), University of Fort Hare, Private Bag X1314, Alice 5700, South Africa; \\ Emeyer@ufh.ac.za \\ * Correspondence: gosayemwenre@ufh.ac.za; Tel.: +27-785-241-528
}

Received: 12 March 2020; Accepted: 9 April 2020; Published: 10 August 2020

\begin{abstract}
This work examines the degradation of photovoltaic modules. It assesses the structural defects of amorphous silicon solar cells, which result from mechanical stress at nanoscale level. Firstly, it analyses the interface morphology, deformation, and internal delamination of a single junction amorphous silicon solar module. Secondly, it explores the interface deformation of the layers of the defective region of the module with some statistical tools including root mean root (RSM) and arithmetic mean (Rq). It used the aforementioned tools to demonstrate the effect of microstructural defects on the mechanical behaviour of the entire layers of the module. The study established that the defect observed in the module, emanated from long-term degradation of the a-Si solar cells after years of exposure to various light and temperature conditions. It tested the mechanism of mechanical degradation and its effect on the reliability and stability of the defective and non-defective regions of the module with adhesion force characterisation.
\end{abstract}

Keywords: scanning probe microscopy (SPM); single junction amorphous silicon solar cell module (a-Si:H); interfaces; $\mathrm{p}-\mathrm{i}-\mathrm{n}$ junction; defective and non-defective samples

\section{Introduction}

The contribution of photovoltaic (PV) electrical energy generation to lowering global energy demands from fossil fuels could increase if their production and cost remain cost-effective. This is important for the reduction of global warming and lowering of carbon dioxide $\left(\mathrm{CO}_{2}\right)$ emissions by $50 \%$ in the next 30 years [1-3]. Cost of production depends on how well a technology has developed. The more a PV technology develops, the less expensive it becomes. This is seen in the recent rapid decrease in the cost of production of monocrystalline based photovoltaic cells compared to how it was 20 years ago [4,5]. It is also crucial to encourage more production and the use of photovoltaic modules, especially in rural areas and areas without grid connections [6]. The above suggestions could make the photovoltaic module a more attractive source of renewable energy. To achieve this, there is a need to improve on the performance of the amorphous silicon module since it is relatively low in hot and humid weather. Currently, the crystalline module is the most dominating PV module. According to Ogbomo et al. [6,7], apart from the stability advantage of the crystalline module, its efficiency is currently $25 \%$. Hence, the need for more research to unravel the real cause of failure and mechanical defects of a-Si:H modules. This would enhance the possibility of designing better a-Si:H modules with high resistance to mechanical degradation, developing and producing next generation photovoltaic modules [7-10]. The elongation of the lifespan of Amorphous Silicon Module is another reason to increase research on the module. Thus, this study will provide an in-depth knowledge on the mechanical properties of degradation and the deformation observed on the a-Si:H samples. The theory behind the analysis in this paper is that, when the different regions on a PV module are analysed, the non-defective region will show low degradation features as opposed to the defective region. This 
is because during operation, the non-defective region shows a low degradation unlike the defective region. This study also presents an investigation of the influence of inter-layer defects on a-Si:H cell stress and mechanical properties deterioration. The cell active layer had a large cross-sectional area of about $1.8 \mu \mathrm{m}$ which reveals the poor quality of the module. Most standard a-Si:H modules have active layers of less than $500 \mathrm{~nm}[3,10]$. Arguably, this large thickness of the active region influences the mechanical stability of the regions in the module.

\section{Materials and Methods}

\subsection{Sample Preparation}

There are different methods of preparing samples for scanning probe microscope (SPM) characterisation including mechanical or cold polishing, cleaving, Focused Ion Beam cutting, and nitrogen assisted fracturing of samples. The methods used depend on the nature of the sample under investigation. For this study, the first stage of the SPM experiment was sample preparation for effective characterisation; the samples were cleaved from a section of the solar cell of interest. Each cleaved sample was polished mechanically by applying diamond-grinding disks before cold polishing was done to reduce the surface roughness from the $10 \mu \mathrm{m}$ to about $10 \mathrm{~nm}$.

\subsection{Towards Reaching the $p-S i: H / i-S i: H / n-S i: H$ Interfaces}

The single junction amorphous silicon solar cell is a difficult material to investigate because of its thin layers [11-13]. The analytical method employed in reaching each of the interface was repeated to get the best measurement. This method is complex because the atomic force microscopy (AFM) tip needs to reach each interface without touching the Ag and Al back contacts, which lie at the end of the cleaved sample. If the cantilever go outside the sample it could cause the tip of the AFM to fall off, reverse, and make the scanning impossible, because the piezo-tube of the KPFM will lose the required feedback. Figure 1 shows the approach that was used to access the different interfaces. The red box shows the AFM tip landing area.

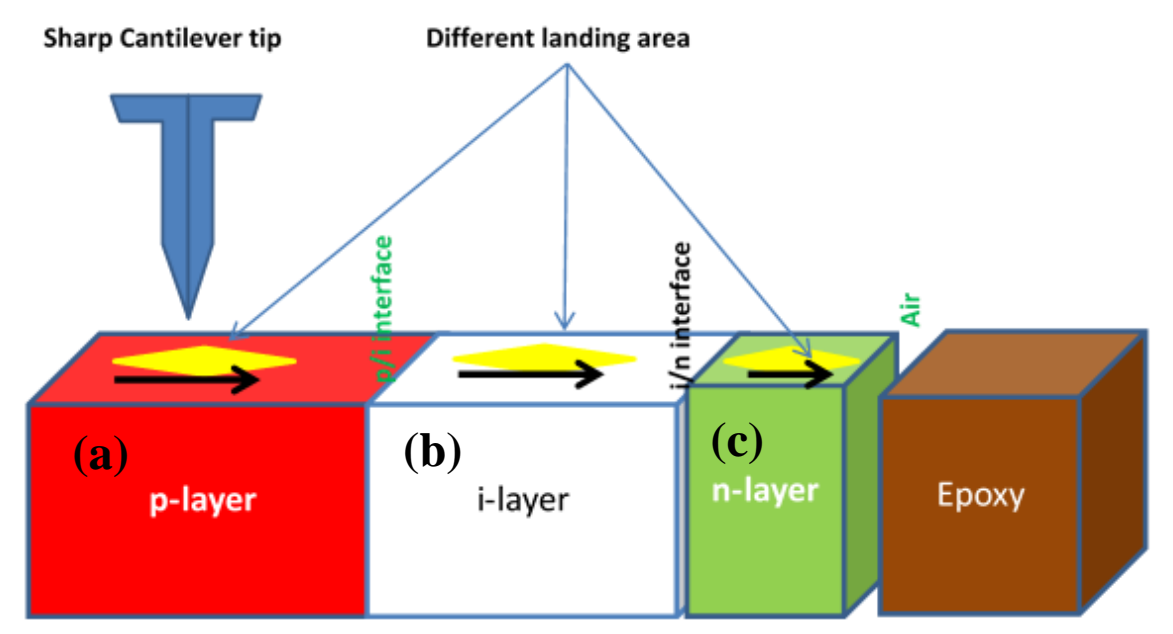

Figure 1. Schematic diagram depicting the different approaches used in investigating the various interfaces: (a) cantilever tip engaging the bulk of the p-Si region, (b) tip progressively approaching the p-Si:H/i-Si:H interfaces and the last stage, (c) tip moving to the last interface, which is i-Si:H/n-Si:H.

To scan, an AFM tip was used to engage the cross-sectional area of the a-Si:H and was moved across the area. The cantilever tip continues to move towards the interface from the bulk side until the Al back contact is observed. The maximum distance that can be engaged for a single scan is $20 \mu \mathrm{m}$. This means that from the glass side to the last interface, five scans have to be done. Hence, enough time is required to do a full scan. For a slow scan, two scans across the bulk sample can last up to $8 \mathrm{~h}$. This length of time is necessary because the exact position of the active layer of the single junction 
amorphous module grown on a glass substrate needs to be ascertained. Once the active region is identified, confocal scanning is performed on the samples. For mechanical parameter measurements at Nano-scale using conducting probe, three extensions are possible and these are electrostatic, current, and impedance. However, for this study, the electrostatic extension map was preferred since it is possible to map the electrostatic force between the delaminated samples and the probing tip. In this investigation, a feedback loop was used to monitor the AFM cantilever amplitude. All measurements were done in the tapping mode as the cantilever tip gently oscillated across the cross-sectional area of the measured samples. Hence, little or no tip was worn-out; this proves that no factor would limit the measurements $[9,14]$. During the measurements, a concise effect was made to ensure that the tip of the cantilever does not exceed the degradation frame of its tip coating. Quality check was done on the AFM cantilever tip by performing a scanning electron microscope (SEM) before and after the measurements to ensure the integrity of the tip.

\subsection{Identification of Interfacial Locations Using Force Quantitative Nano-Mechanical (PF-QNM)}

The analysis of basic adhesion mechanisms at nanoscale for amorphous silicon solar cells would be of substantial interest to photovoltaic (PV) researchers in the near future. The bulk of this work was motivated by the need to enhance the comprehension of the relationship between electrical degradation and mechanical degradation in the a-Si:H $[15,16]$. An in-depth understanding of the adhesion degradation of an a-Si:H on a nano-scale seeks to provide systematic guidelines for the a-Si:H thin film research and design [16-18]. To ensure that the evaluated adhesions presented here are the real values of the intrinsic layers of the a-Si:H samples, a successive slow scan of the cross-sectional area in modulating peak force mode was performed. Note that any SPM technique that employs AFM as a quantitative diagnostic tool in analysing interfacial adhesion requires calibration procedures. Thus, the cantilever tip was calibrated according to the standard procedure and a non-contact mechanic modelling was used throughout the process. This was done to prevent any significant change in the adhesion data acquired as a result of the subtle nature of its environmental conditions. The process was monitored closely throughout the duration of the measurement. The setup excluded any surface contamination or modification. All the images of AFM tips used were with a high-resolution scanning electron microscope (SEM) before and after the measurement, to check for any tip modification that could alter the pull-off force of the cantilever tip. This technique is automatically able to calculate the pull-off force from the force-distance plot using the calibration procedure before the measurement $[19,20]$. To determine the appropriate position of the region of interest, an extremely low value was used for the elastic constant and equilibrium spacing. As the tip approached the interface, the pull-off force drastically decreased. This process was used to identify the interface and acquire the data. In this Peak Force Quantitative Nano-mechanical (PF-QNM) mode, the device calculated the adhesion values from the pull-of force data acquired using the equation presented below. Derjaguin Muller and Toporov (DMT) gave a direct expression for adhesion by assuming a constant deformation as:

$$
\mathrm{a}=\left\{\frac{\mathrm{R}}{\mathrm{K}}(\mathrm{P}+2 \pi \gamma \mathrm{R})\right\}^{1 / 3}
$$

where a is the contact area: this is zero as the pull-off force due to a negligible contact stress, $R$ is the radius of the sphere, $\mathrm{P}$ is the pull-off force, and $\gamma$ is the adhesion work. Hence, by rearranging Equation (1), adhesion work is directly evaluated as:

$$
\begin{aligned}
& \mathrm{P}_{\mathrm{C}(\mathrm{DMT})}=-2 \pi \gamma \mathrm{R} \\
& \lambda=2 \sigma_{0}\left(\frac{\mathrm{R}}{\pi \gamma \mathrm{K}^{2}}\right)^{1 / 3}
\end{aligned}
$$


where $\sigma_{0}$ is constant adhesion stress.

The investigation of mechanical degradation in the a-Si:H material using PeakForce QNM provides in-depth information regarding the material property [21]. Such knowledge enhances the understanding of mechanical properties of degraded a-Si:H samples.

\subsection{Identification of Various Interfaces}

The exact location and position of the active layer is an issue in a study of this nature; hence, the first step is to identify the positions of the p-layer, i-layer and n-layer. In Figure 2, the Derjaguin Muller and Toporov (DMT) Modulus's measurement of the non-affected region is presented. The DMT image provides better images for obtaining the positions of the various layers. This is the most reliable technique that creates an understanding that the rest of the characterisations of the various mechanical properties of the single junction amorphous module were acquired from this region of interest. This procedure was important in avoiding misinterpretation of results.

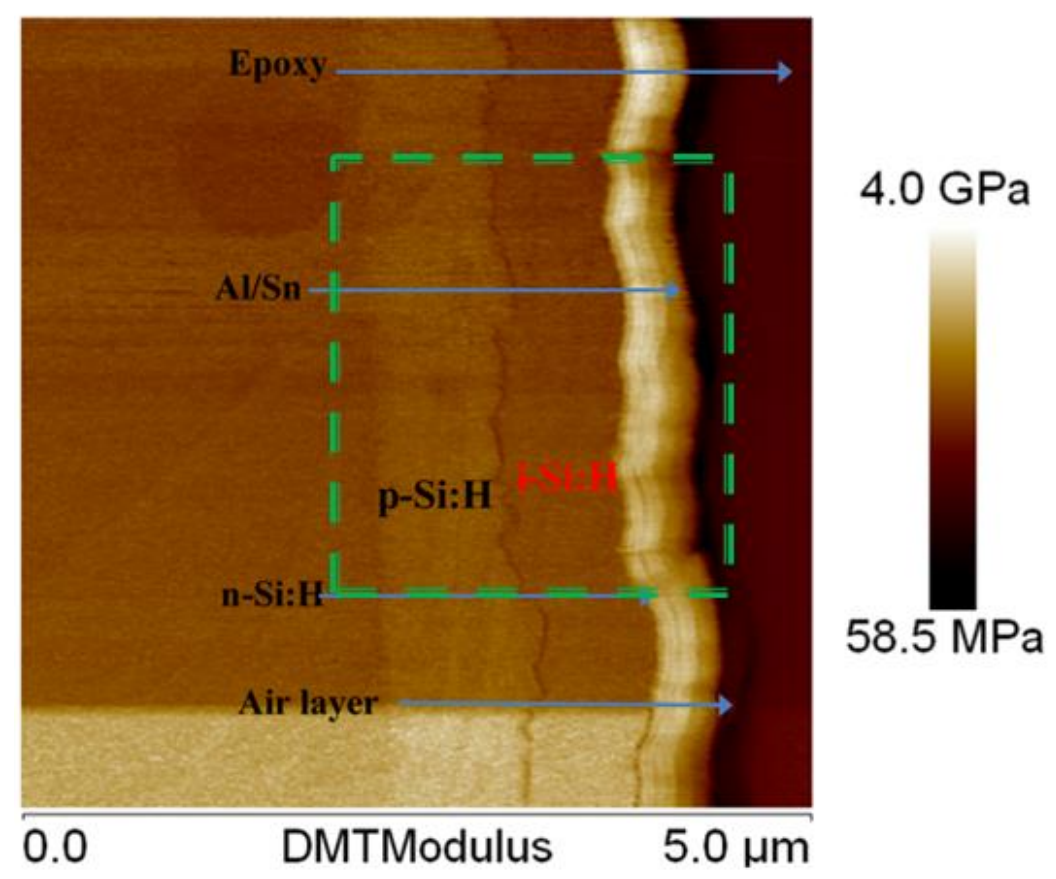

Figure 2. Shows the Derjaguin Muller and Toporov (DMT) Modulus of a sample from the non-defective region of the a-Si:H under investigation.

From Figure 2, we can observe four interfaces, the last of which is the one separating the Al contact from the residual of the plastic back cover. Although the plastic back cover was carefully removed from each sample, the PFQNM scan was still able to pick the thin layer that was not visible in the normal KPFM scan. Another importance of the DMT modulus mapping is that it provides the reduced Young's modulus of elasticity; hence the Young's modulus of the solar material can also be calculated if necessary. Having identified the various interfaces for a detailed investigation of adhesion, a region in Figure 2 was marked out for analysis, as indicated by the green box.

\section{Results}

\subsection{Adhesion}

To measure the adhesion between the layers, a sample size of $20 \mu \mathrm{m}$ was scanned at a rate of $0.701 \mathrm{~Hz}$. The sample line was 512 for a retraced line direction and the mapping was captured in an upward direction. This was done throughout to achieve uniformity in the results. The probing tip had an amplitude set point of $250 \mathrm{mV}$, while the driving amplitude was $15.26 \mathrm{mV}$ throughout the 
measurements. Although the slow scan is time consuming, it is encouraged in order to account for every single point in the nano-scale measurement. PeakForce Quantitative Nano-mechanical Mapping (QNM) was used to simultaneously probe the morphology and the mechanical properties [12,21] to study the adhesive strength between interface layers. The technique is illustrated by the famous distance-force curve. The curve shows a point to point fitting of the curve with the aid of the Derjaguin Muller and Toporov (DMT) model of elastic contact [14,16,21]. In this study, a special Atomic force microscopy (AFM) probing tip that had been calibrated was used to gain insight into the binding force existing between each interface. From the surface coverage acquired and considering the nature of the a-Si:H, the layers of which are very thin, much difference should not be expected. In other words, a small difference can be used to arrive at an accurate conclusion. The morphological properties of both samples from the defective and non-defective regions were examined and their results demonstrated variations in their roughness. Figure 3 shows the adhesion mapping of the samples, but for analysis, only the region marked out in Figure $3 a$ is used to investigate the adhesion force of the active layer. Since the area scanned in Figure $3 a$ is larger than the region of interest, which is the active region, the area marked in Figure 3a was scanned for a further analysis as shown in Figure 4. Figure 4a is a 2-D image of the adhesion force, while Figure $4 b$, which is a 3-D image shows where the adhesion force mapping was acquired and illustrates the positions of the p-Si:H/i-Si:H, i-Si:H/n-Si:H, and the n-Si:H/Ag interfaces. Although the n-Si:H/Ag is not so pronounced, the adhesion curve clearly shows the presence of the different interfaces.

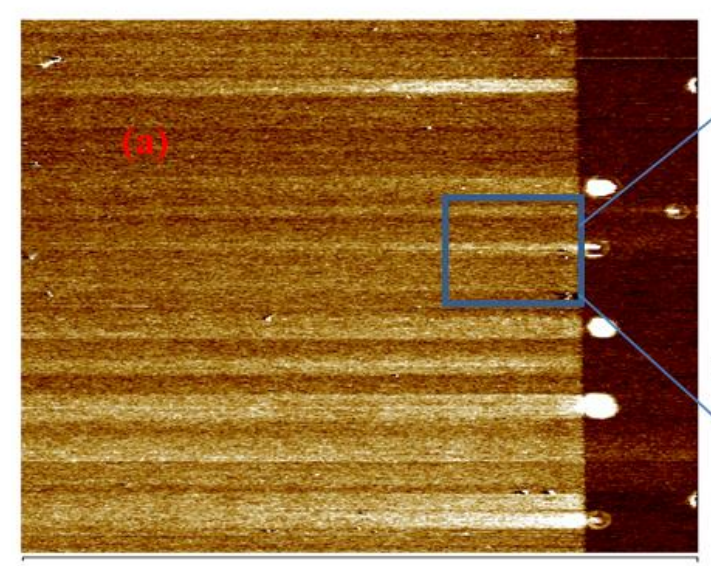

0.0

Adhesion

$20.0 \mu \mathrm{m}$

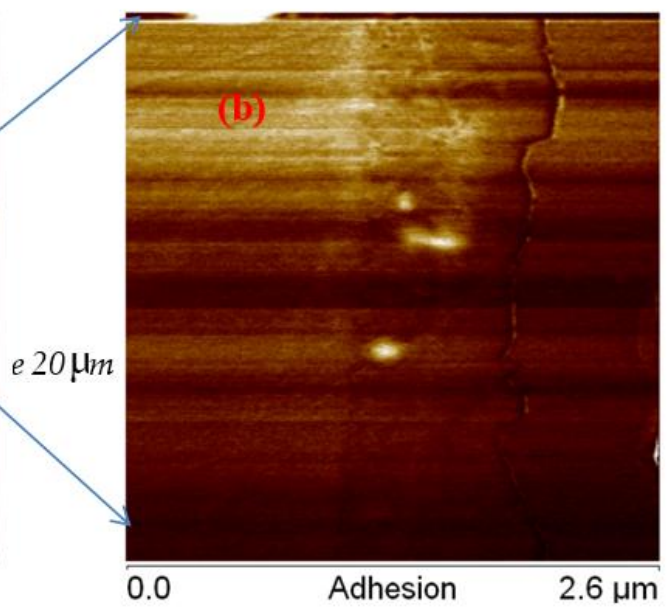

$31.2 \mathrm{nN}$

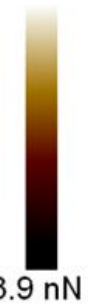

$3.9 \mathrm{nN}$

Figure 3. Adhesion images of sample: (a) size $20 \mu \mathrm{m} \times 20 \mu \mathrm{m}$. (b) size $2.6 \mu \mathrm{m} \times 2.6 \mu \mathrm{m}$. A cross-sectional view of the sample showing (a) the maximum scanned area, (b) the region of interest (active region) of the non-defective sample.
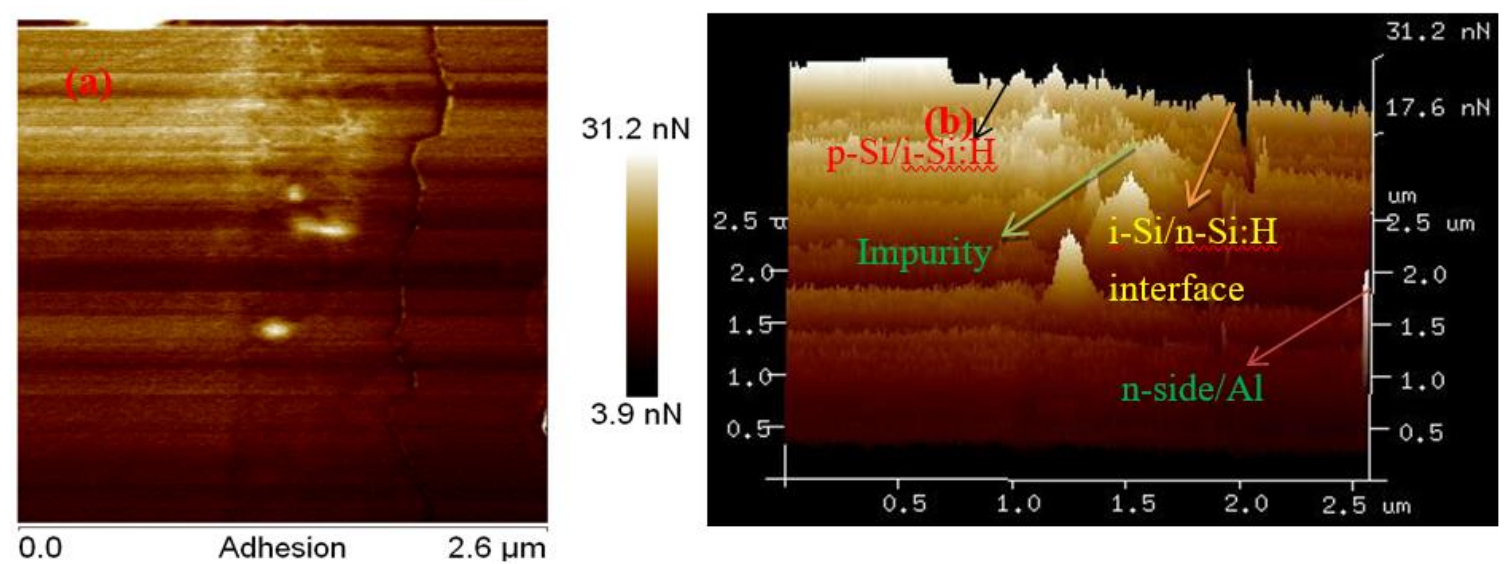

Figure 4. A cross-sectional view of the adhesion map for the non-defective sample of the a-Si:H as indicated in Figure 3. (a) 2-D image of the sample and (b) 3-D image of the sample. 
Though the various interfaces are indicated, the p-Si:H/i-Si:H and n-Si:H are the most prominent ones. In Figure $4 b$, it is clear that there is a gradual decline in the adhesion force from the p-Si:H to $\mathrm{n}$-Si:H region. Furthermore, peaks are also observed and this is an evidence of foreign (impurity) elements in the intrinsic layer. The position of the various interfaces can also be identified from the graph of adhesion against a cross-sectional distance. Similarly, the Derjaguin Muller and Toporov (DMT) Modulus measurement of the affected region is presented in Figure 5. The DMT image provides a clear picture of the transformation that occurred in the defective region due to hotspot formation.

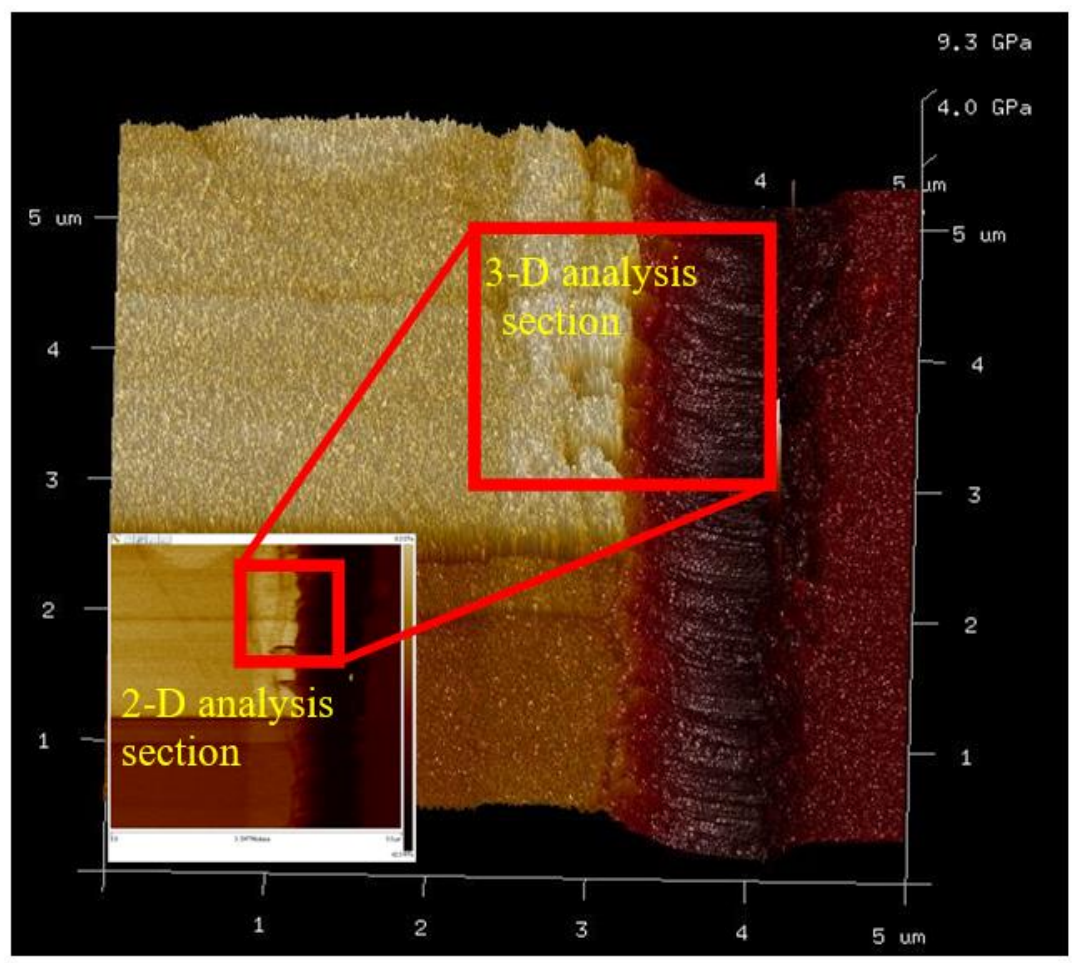

Figure 5. Shows the DMT Modulus of a sample from the defective region of the a-Si:H sample under investigation.

From Figure 5, only three interfaces are visible and the last interface separating the Al contact from the residual plastic back cover is seen to have collapsed due to structural damage. The observed structural deformation is linked to a hot spot formation. Figure 6 presents the measurement of the adhesion force of the affected sample. The blue box in Figure $6 \mathrm{a}-\mathrm{c}$ indicates the region of interest. Figure $6 \mathrm{~b}$ is characterised by a high value of the layer of the epoxy after the back contact. This influenced the value of the adhesion force seen on the image around the back contact due to high quantities of the epoxy layer. Image-mapping presentation is not the best way to quantify mechanical properties (adhesion force) for the following reasons: firstly, the image scale depends on other factors apart from the intrinsic properties of the material, such as title angle and the colour code that was used. Secondly, it can be affected by the modification in the software after series of scans have been done. Therefore, the only reliable way of quantifying material properties is through the data obtained during scanning.

For a comprehensive analysis of the adhesive force measurements, the data acquired from each scan are analysed. The measured data for the adhesion force of different samples from the defective and non-defective regions are presented in Figure 7. 


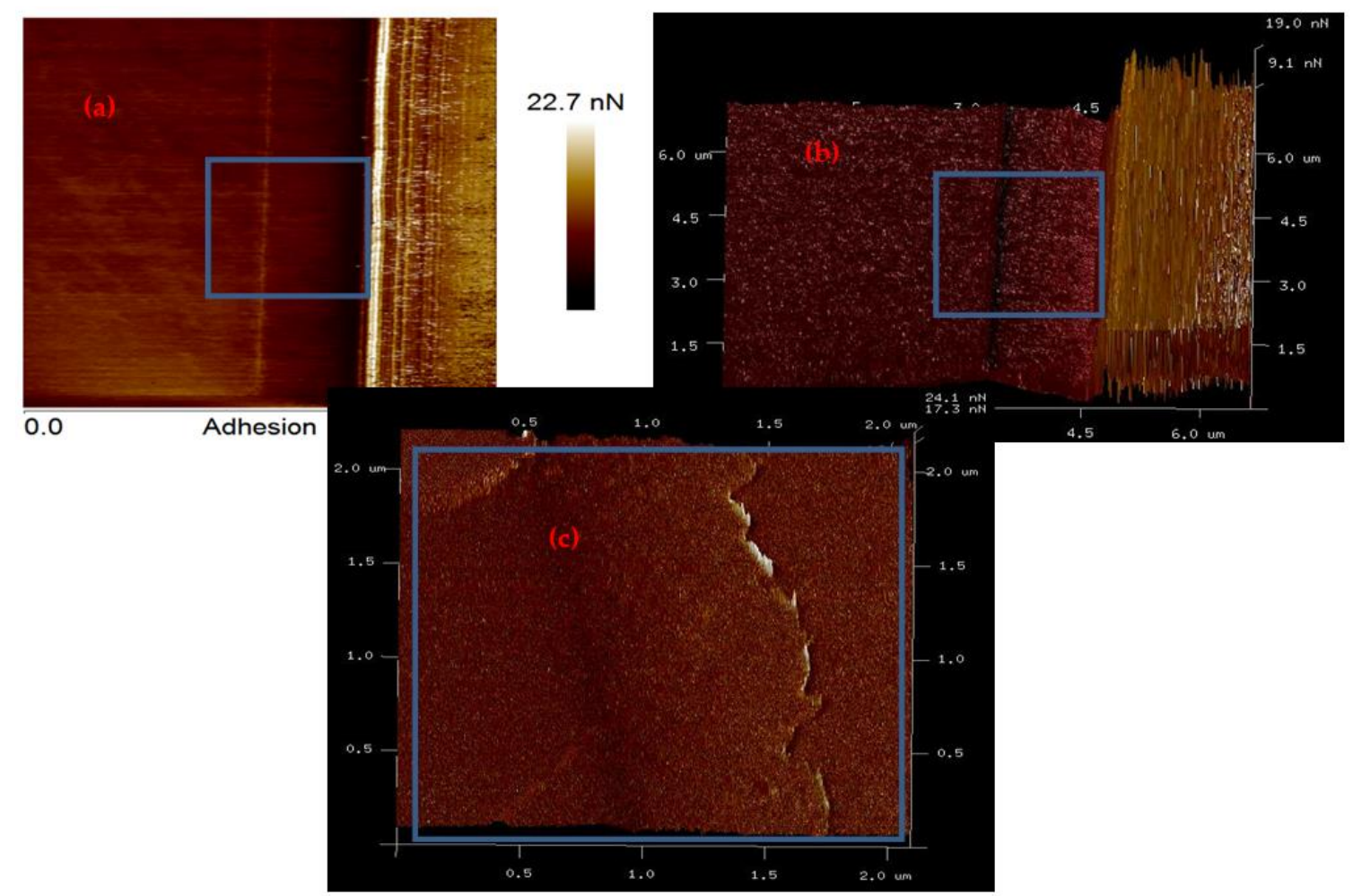

Figure 6. Adhesion map for defective sample of a-Si:H, the scanned area is $2.6 \mu \mathrm{m} \times 2.6 \mu \mathrm{m}$. (a) 2-D image, (b) 3-D image showing the epoxy region and (c) 3-D image showing the area of interest.

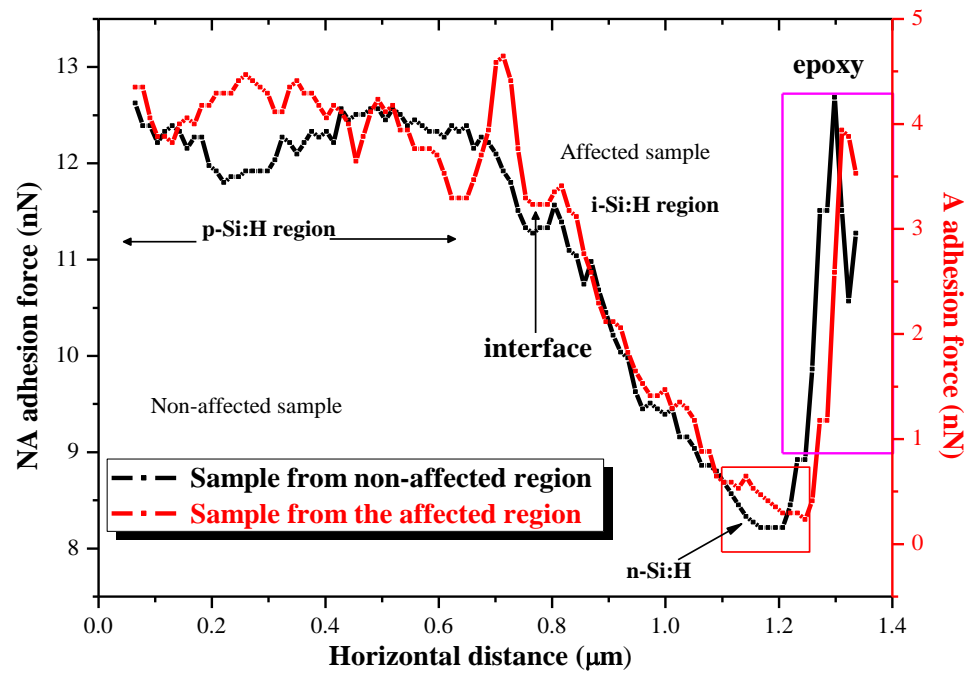

Figure 7. Adhesion force of the sample from the defective region and the non-defective region close to discoloured region.

The most dominate interface in Figure 7 include glass/p-Si:H, p-Si:H/i-Si:H, i-Si:H/n-Si:H n-Si:H/air. In Figure 7, the adhesion force at the glass side of the p-Si:H interface was much higher than the other regions, but it decreased as it moved away from the p-layer until it reached the $p / i$ interface. For the non-defective sample, the adhesion force of the n-layer was approximately $8.2 \mathrm{nN}$ and that of the p-layer was $12.7 \mathrm{nN}$. The average adhesion force in the p-layer of the non-defective sample was $12.2 \mathrm{nN}$. However, after the first interface, which was about $800 \mathrm{~nm}$, the adhesive force began to decline steadily until it reached the n-layer. The adhesion force of the sample from the defective region (red) appeared constant inside the p-layer and had a sharp rise at about $715 \mathrm{~nm}$ distance before the first interface. The adhesive force steadily decreased inside the intrinsic layer until it got to the 
n-layer where it maintained some uniformity again, before it started to rise again. The steady rise in the adhesion force after the n-layer was due to the presence of plastic particles and epoxy that were not part of the solar material. For the red curve (defective sample), the adhesion force at the p-layer was approximately $4.2 \mathrm{nN}$ as opposed to the non-defective sample, which was $12.7 \mathrm{nN}$. The sharp increase as shown in the curve close to the first interface (p-Si:H/i-Si:H) of defective sample in Figure 7 is the result of foreign elements that had a high binding force. Additionally, at the thin n-layer, an average adhesion force of $0.24 \mathrm{nN}$ was recorded for the defective sample; this is much lower than the $8.2 \mathrm{nN}$ recorded for the non-defective sample. The adhesion degradation demonstrated above is unhealthy for a-Si:H solar module in terms of its output and durability. Adhesion degradation impacted negatively on the ability of a material to absorb photon energy; this is documented by Drijkoningen [14,22]. In addition, adhesion degradation between various layers affects interface binding energy and aids the delamination of the layers of solar cells. This can lead to low output and ultimately result in premature failure as reported in previous study by Osayemwenre's @ et al., 2019. In that study in 7 years since the beginning of the monitoring process, two modules were observed to have failed [20-22]. Additional measurements were taken for the defective and non-defective samples at the intrinsic region, starting from the $\mathrm{p} / \mathrm{i}$ interface to $\mathrm{i} / \mathrm{n}$ interface. The results as shown in Figure 8 below reveal that the n-layer of the defective sample is about $30 \mathrm{~nm}$ shorter than that of the non-defective sample.
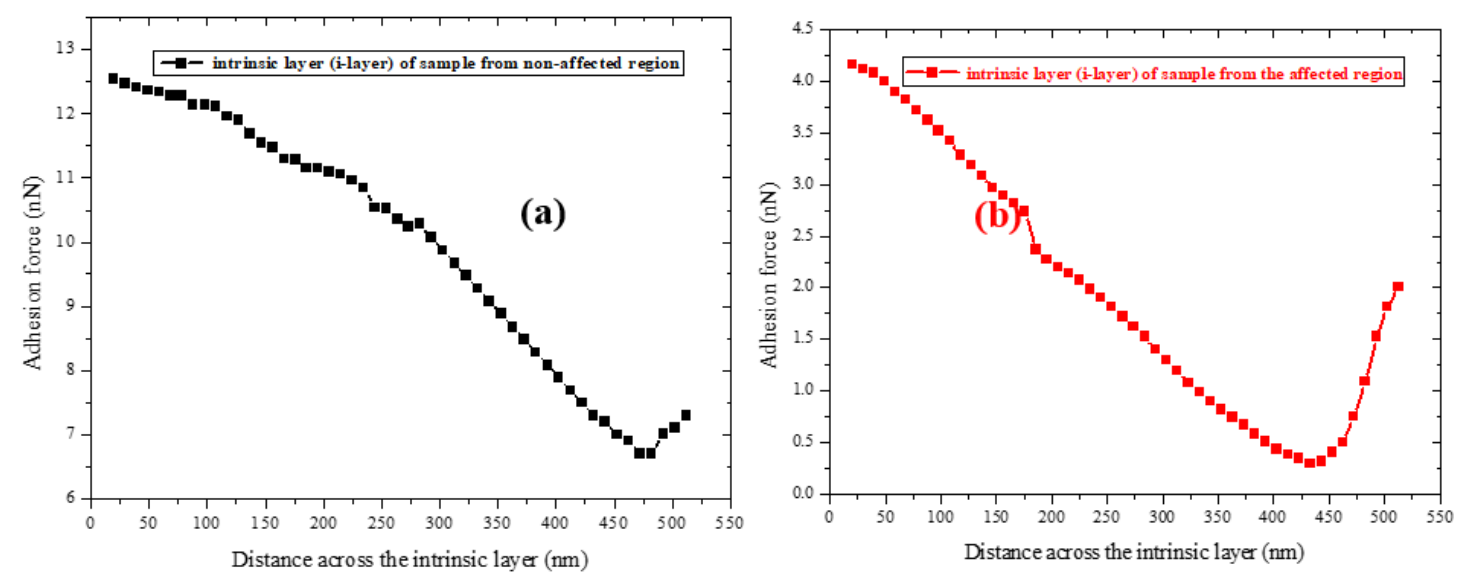

Figure 8. Adhesion force acquired from a distance immediately after the $\mathrm{p} / \mathrm{i}$ to $\mathrm{i} / \mathrm{n}$ interfaces for: (a) non-defective sample, (b) defective sample. The increase in adhesive force after $500 \mathrm{~nm}$ represents the epoxy layer, which is very sticky in nature.

The adhesion curve presented in Figure 8a has a maximum adhesion force of $12.6 \mathrm{nN}$ and a minimum adhesive force of $7.6 \mathrm{nN}$. The adhesion force various inside the i-layer of the non-defective sample is $40 \%$. This means that enough adhesion force exists between the p-layer interfaces and the n-layer interface in the non-defective region. However, in Figure $8 b$, the maximum value of the adhesion force is $4.4 \mathrm{nN}$ while the least is $0.24 \mathrm{nN}$ and this translates to a $94 \%$ various between the p-layer and n-layer interfaces in the defective region. This implies that the adhesive force binding the layers together is weak and so the region is a good channel for excessive water ingress or impurity.

\subsection{Deformation}

The effect of adhesion degradation on the layers of the a-Si:H module was investigated through its cross section. To this effect, the deformation experienced by the sample from the defective and non-defective regions were analysed to establish the relationship between their mechanical properties. The results of both samples are presented in Figure 9.

In both samples, the p-layers experienced steady deformation until they reached a distance of about $0.76 \mu \mathrm{m}$ mark from the $\mathrm{p} / \mathrm{i}$ interface where they maintained a brief uniformity. For the non-defective region, the sample maintained a uniform deformation inside the i-layer, meaning that the 
region experienced a negligible deformation due to the absence of structural damage. However, for the defective region, the sample continued to experience deformation throughout the i-layer. This means that the force binding the various layers was weak. This observation is linked to the presence of hotspot formation which can lead to delamination of layers. In the non-affected sample, the maximum deformation was $1.4 \mathrm{~nm}$ while the minimum was $7.8 \mathrm{~nm}$ at the $\mathrm{n}$-layer. However, for the affected sample, the maximum deformation was $21.4 \mathrm{~nm}$, while the minimum is $15.0 \mathrm{~nm}$ in the n-layer. Another observation was that the epoxy had relatively negligible deformation and was close to the value of the n-layer in both samples.

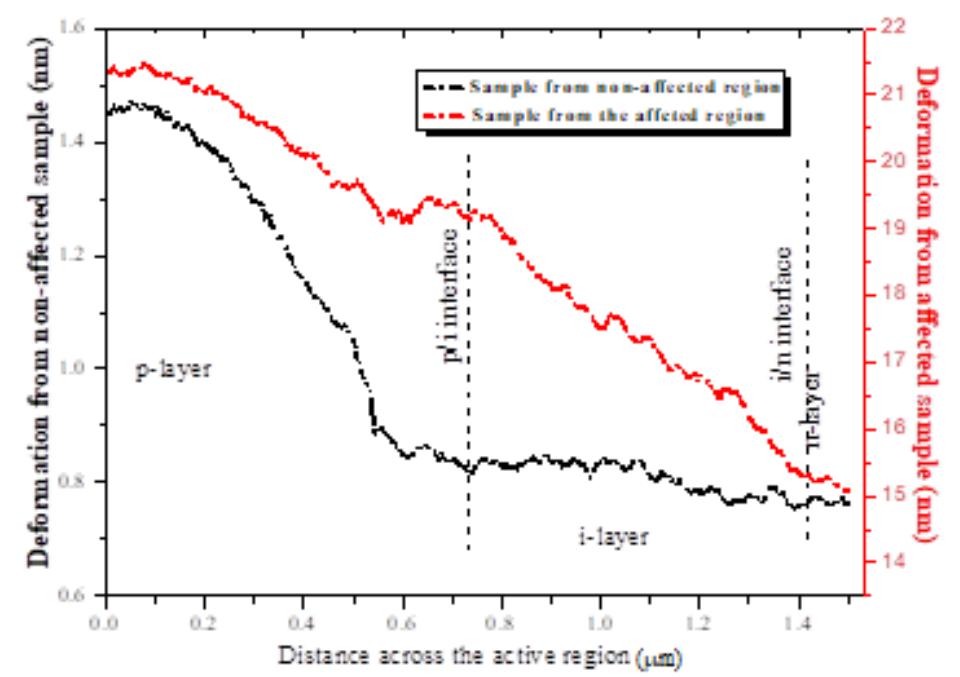

Figure 9. Deformation investigation of the sample from the non-defective region (black) and the sample from defective region (red).

\subsection{Deformation and Morphology Analysis}

Deformation analysis was done using statistical parameters to illustrate the relationship between the adhesion force and the observed deformation. In this case, the affected region of the module has a weaker bond in between its interfaces; this explains why some chemical particles, in some cases, can easily migrate, leading to inter-chemical diffusion. Once this happens, the mechanical strength existing between different layers decreases; resulting in a softer region than the non-affected region. This softer region will exhibit a higher degree of damage. Therefore, the affected region has a higher deformation, as can be seen in Table 1 below. It is mostly believed that the degree of sample deformation is related to sample indentation by the cantilever tip, but this is generally not true because deformation variations can also occur in samples of equal hardness $[15,23,24]$. All the data acquired for deformation analysis were positive, since negative data connote the falling off of the AFM tip, therefore, deformation should not be negative.

Table 1. Effect of deformation on samples morphology.

\begin{tabular}{ccccccc}
\hline Sample & $\begin{array}{c}\text { Horiz Dist } \\
(\boldsymbol{\mu m})\end{array}$ & $\begin{array}{c}\text { Vertical Dist } \\
(\mathbf{n m})\end{array}$ & $\begin{array}{c}\text { Surface Dist } \\
(\boldsymbol{\mu m})\end{array}$ & $\begin{array}{c}\text { RmaxRa } \\
(\mathbf{n m})\end{array}$ & $\begin{array}{c}\text { RMS Rq } \\
(\mathbf{n m})\end{array}$ & $\begin{array}{c}\text { Av Height } \\
(\mathbf{R a})(\mathbf{n m})\end{array}$ \\
\hline Affected & 1.243 & -3.265 & 0.265 & 10.091 & 1.455 & 2.895 \\
Non-affected & 1.243 & -0.465 & 0.201 & 5.202 & 0.571 & 1.402 \\
Non-affected (BR) & 1.233 & -0.408 & 0.218 & 6.513 & 0.129 & 1.457 \\
\hline
\end{tabular}

The net adhesion force that exists between the different layers and interfaces in the a-Si:H solar cells is the contribution of every single molecule present in different layers involved in the binding process. The deformation measurements were done within the active region for both the affected and non-affected sample and the result is presented in Table 1. The scans display the average roughness 
$(R a)$ of $2.89 \mathrm{~nm}$ for the affected. For the non-affected sample, an average roughness $(R a)$ of $1.457 \mathrm{~nm}$ was measured for the sample with brown colouration and $1.402 \mathrm{~nm}$ for the samples far from the region with a brown colour. The RMS roughness for the affected sample is $1.455 \mathrm{~nm}$ while the RMS for the non-affected sample close to the region with a brown colour (BR) is 0.612 and the RMS for the non-affected sample, far from the discoloration region, is $0.571 \mathrm{~nm}$. The rationale behind using two non-affected samples was to see if mechanical degradation takes place at the regions that are prone to ethyl vinyl acetate (EVA) degradation and compare the result with the one from the region not close to EVA discolouration.

\section{Conclusions}

This study used Scanning Probe Microscope to investigate the effect of the degradation which resulted from a defect on the mechanical properties of a single junction amorphous silicon module. It examined these mechanical properties across the interface at nanoscale level by analysing the adhesive force and deformation between the various layers in the active region. The study found that the root mean square (RMS) roughness of the sample from the defective and non-defective regions were different from both the localised and net measurements taken. This was as a result of the difference in the defect level across the module. It also found that the estimated interface roughness increased because of defects. Furthermore, the study reveals the need to employ other mechanical parameters to assess the mechanical properties of a-Si:H modules by using all the dynamic AFM modes which represent the material properties of the $p-i-n$ solar cells. Some of these include inphase, phase, quadrature, and DMT Modules, although these cannot be used conclusively on their own to represent the mechanical properties of the analysed samples. Notwithstanding, it is one characterisation technique that can be used to substantiate the variation and quality of a-Si:H materials. In addition, phase image in AFM modes represents the mechanical properties of the material and some mechanical properties of the solar cells can be calculated from the output of the device lock-in amplifier. The average value of the curve (Figure 7) obtained from the local mechanical properties in the defective and non-defective sample was used to estimate the degree of degradation of the module. In addition, the variation in the RMS values of the sample is the effect of the inhomogeneity of the mechanical properties distribution as a result of degradation. This might have caused the inter-chemical diffusion which occurred at some regions of the defective sample.

Author Contributions: G.O. conceived and wrote the paper; E.M. contributed analysis tools; E.M. assisted in the design of the experiments; G.O. proposed the theory and analysed the experimental data. All authors have read and agreed to the published version of the manuscript.

Funding: This research received no external funding.

Acknowledgments: The authors would like to express their gratitude to the following organization: University of Fort Hare and Govan Mbeki Research \& Development Centre (GMRDC and the financial contribution of NRF, DSI.

Conflicts of Interest: The authors declare no conflict of interest.

\section{References}

1. UN Environment and International Energy Agency. Towards a Zero-Emission, Efficient, and Resilient Buildings and Construction Sector; Global Status Report; UN Environment and International Energy Agency: Paris, France, 2017.

2. IEA World Energy Outlook 2019. Available online: https://www.iea.org/reports/world-energy-outlook-2019 (accessed on 10 February 2020).

3. Almosni, S.; Delamarre, A.; Jehl, Z.; Suchet, D.; Cojocaru, L.; Giteau, M.; Behaghel, B.; Julian, A.; Ibrahim, C.; Tatry, L.; et al. Material challenges for solar cells in the twenty-first century: Directions in emerging technologies. Sci. Technol. Adv. Mater. 2018, 19, 336-369. [CrossRef] [PubMed]

4. Louwen, A.; van Sark, W.G.J.H.M.; Faaij, A.P.C.; Schropp, R.E.I. Re-assessment of net energy production and greenhouse gas emissions avoidance after 40 years of photovoltaics development. Nat. Commun. 2016, 7, 13728. [CrossRef] [PubMed] 
5. Department of Energy. Photovoltaic System Pricing Trends: Historical, Recent, and Near-Term Projections, 2014th ed.; Department of Energy: Washington, DC, USA, 2014.

6. Ogbomo, O.O.; Amalu, E.H.; Ekere, N.N.; Olagbegi, P.O. A review of photovoltaic module technologies for increased performance in tropical climate. Renew. Sustain. Energy Rev. 2017, 75, 1225-1238. [CrossRef]

7. Paggi, M.; Berardone, I.; Infuso, A.; Corrado, M. Fatigue degradation and electric recovery in silicon solar cells embedded in photovoltaic modules. Sci. Rep. 2014, 4, 4506-4512. [CrossRef] [PubMed]

8. Paggi, M.; Kajari-Schröder, S.; Eitner, U. Thermomechanical deformations in photovoltaic laminates. J. Strain Anal. Eng. Des. 2011, 46, 772-782. [CrossRef]

9. Eitner, U. Thermomechanics of Photovoltaic Modules. Ph.D. Thesis, Martin Luther University, Halle-Wittenberg, Germany, 2011.

10. Koppelaar, R.H.E.M. Solar-PV energy payback and net energy: Meta-assessment of study quality, reproducibility, and results harmonization. Renew. Sustain. Energy Rev. 2017, 72, 1241-1255. [CrossRef]

11. Knoll, A.; Horvat, A.; Lyakhova, K.S.; Krausch, G.; Sevink, G.J.A.; Zvelindovsky, A.V.; Magerle, R. Phase behavior in thin films of cylinder forming block copolymers. Phys. Rev. Lett. 2002, 89, 035501. [CrossRef] [PubMed]

12. Sader, J.E.I.; Sanelli, J.A.; Adamson, B.D.; Monty, J.P.; Wei, X.; Crawford, S.A.; Friend, J.R.; Marusic, I.; Mulvaney, P.; Bieske, E.J. Spring constant calibration of atomic force microscope cantilevers of arbitrary shape. Rev. Sci. Instrum. 2012, 83, 103705. [CrossRef] [PubMed]

13. Shikler, A.; Meoded, T.; Fried, N.; Rosenwaks, Y. Potential imaging of operating light-emitting devices using kelvin force microscopy. Appl. Phys. Lett. 1999, 74, 2972-2974. [CrossRef]

14. Su, C.; Huang, L.; Neilson, P.; Kelley, V. In-situ measurements of in plane and out-of-plane force gradient with a torsional resonance mode afm. In Proceedings of the 12th International Conference on Scanning Tunnelling Microscopy/Spectroscopy and Related Techniques, Eindhoven, The Netherlands, 30 December 2003; p. 349.

15. Maboudian, R. Adhesion and friction issues associated with reliable operation of MEMS. Mater. Res. Soc. Bull. 1998, 23, 23-47. [CrossRef]

16. Osayemwenre, G.O.; Meyer, E.L.; Taziwa, R.T.; Mamphweli, S.N. Photothermal degradation of degraded a-Si:H. Ovonic Res. J. 2017, 10, 421-444.

17. Mastrangelo, C.H.; Hsu, C.H. A Simple Experimental Technique for the Measurement of the Work of Adhesion of Microstructures; IEEE Solid-State Sensor Actuator Workshop: Hilton Head, SC, USA, 1992; p. 208.

18. Voigtländer, B. Scanning Probe Microscopy: Atomic Force Microscopy and Scanning Tunneling Microscopy; Springer: Berlin, Germany, 2005; pp. 573-579.

19. Melitz, W.; Shen, J.; Kummel, A.C.; Lee, S. Surface Science Reports Kelvin probe force microscopy and its application. Surf. Sci. Rep. 2011, 66, 1-27. [CrossRef]

20. Osayemwenre, G.O.; Meyer, E.L. Confirmation of the degradation of single junction amorphous silicon modules (a-Si:H). Int. J. Photoenergy 2019, 2019, 3452180. [CrossRef]

21. Barbet, S.; Popoff, M.; Diesinger, H.; Deresmes, D.; Théron, D.; Mélin, T. Cross-talk artefacts in Kelvin probe force microscopy imaging: A comprehensive study. J. Appl. Phys. 2014, 115, 144313. [CrossRef]

22. Sader, E.J. Calibration of rectangular atomic force microscope cantilevers. Rev. Sci. Instrum. 1999, 70, 3967. [CrossRef]

23. Osayemwenre, G.O.; Meyer, E.L.; Taziwa, R.T. Investigation of defect in c-Si solar cells by Confocal Raman Spectroscopy. Energy Environ. J. 2018, 29, 1525-1533. [CrossRef]

24. Sandberg, R.; Boisen, A.; Svendsen, W. Characterization system for resonant micro- and nanocantilevers. Rev. Sci. Instrum. 2005, 76, 125101. [CrossRef]

(C) 2020 by the authors. Licensee MDPI, Basel, Switzerland. This article is an open access article distributed under the terms and conditions of the Creative Commons Attribution (CC BY) license (http://creativecommons.org/licenses/by/4.0/). 